6.Wickwire EM. Value-based sleep and breathing: health economic aspects of obstructive sleep apnea. Fac Rev 2021;10:40. doi: 10.12703/r/10-40

7.Chung F, Yegneswaran B, Liao P, Chung SA, Vairavanathan S, Islam S, et al. STOP questionnaire: A tool to screen patients for obstructive sleep apnea.
Anesthesiology 2008;108: 812-21. doi: 10.1097/ ALN.0b013e31816d83e4

8. H. Marti-Soler, C. Hirotsu, P. Marques-Vidal, et al. The NoSAS score for screening of sleep-disordered breathing: a derivation and validation study. Lancet Respir Med 2016;4:742-748. doi: 10.1016/ S2213-2600(16)30075-3

\title{
Sleep Disorders and Risk of CVD
}

\author{
DAGMARA HERING \\ Department of Hypertension and Diabetology \\ Medical University of Gdansk, Poland, \\ Gdansk, Poland.
}

DOI:10.30824/2106-13

\section{According to Thomas Dekker quote "Sleep is that golden chain that ties health and our bodies together".}

Insufficient and inadequate sleep quality is a growing health problem affecting more than one in three adults. Sleep disorders are critical contributors to obesity, diabetes, cardiovascular (CV) disease, heart failure (HF), stroke and depression. Sleep debt increases the risk of motor vehicle crashes, fatal injury, and disability each year.

Sleep can be disrupted by 'sleep and circadian rhythm disorders' (i.e., sleep apnoea, insomnia, narcolepsy, restless leg syndrome, parasomnias, sleep bruxism), work, social or lifestyle behaviours. Unhealthy sleep patterns defined as short $(<7$ hours) and long sleep ( $>8$ hours), late chronotype, insomnia symptoms, snoring and excessive daytime sleepiness (EDS) have been associated with an increased risk of coronary heart disease (CHD), CV disease, stroke, and all-cause mortality. While patients with a healthy sleep score (Figure 1a) demonstrated a reduced risk of CV disease by $35 \%$, CHD by $34 \%$ and stroke by $34 \%$ independently of genetic risk score ${ }^{1}$, and $\mathrm{HF}^{2}$ suggesting that treating sleep disorders should be considered for $\mathrm{CV}$ prevention.

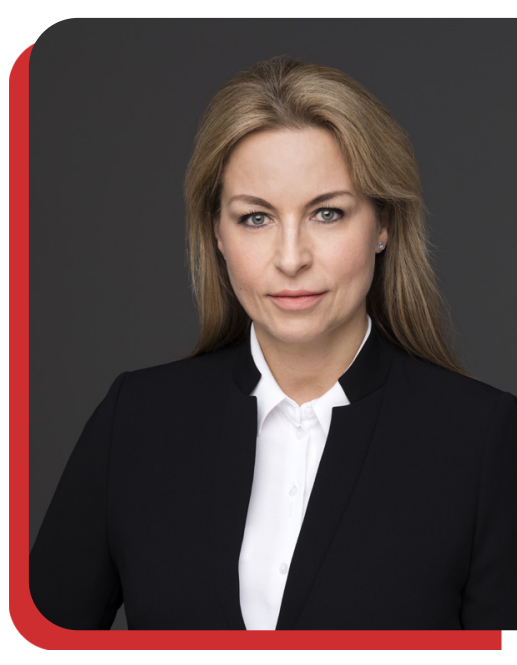

Obstructive sleep apnoea (OSA) is the most common type of sleep-disordered breathing [3]. Nearly 1 billion of the worldwide adults' population aged 30-69 years have OSA, and the number of people with moderate to severe OSA, for which treatment is generally recommended, is estimated 425 million $^{4}$. Given the already high and increasing prevalence of obesity and aging, the number of people presenting with OSA symptoms continues to grow.

OSA is characterized by recurrent episodes of closure (apnoea) and hypopnea (narrowing) of the upper respiratory airflow resulting in shallow or paused breathing (Figure 1b), intermittent hypoxemia, and arousal associated with maintained or increasing ventilator efforts ${ }^{3}$. Apnoea is defined as at least $90 \%$ airflow limitation through the respiratory tract which is maintained for 10 seconds and associated with increased inspiratory efforts from chest and/or abdomen, whereas hypopnea refers to a drop in the airflow of $\geq 30 \%$ for $\geq 10$ seconds and is associated with a $\geq 3 \%$ oxygen desaturation or arousal. Based on the index denoting average number of apnoeic and hypopneic episodes per hour of sleep (apnoeahypopnea-index [AHI], OSA is classified as: mild $\mathrm{AHI} \geq 5$ and $<15$ events per hour, moderate $\mathrm{AHI}$ $\geq 15$ and $\leq 30$ evets per hour, and severe $\mathrm{AHI}>30$ events per hour. 
The most common symptoms, risk factors and OSA associated conditions are summarized in Table.

OSA mediated intermittent hypoxia has been suggested as the critical contributor to increased morbidity and mortality. Evidence is emerging on the casual relationship between OSA and development and management of hypertension, diabetes, cardiac arrythmia, HF, sudden cardiac death (SCD), chronic obstructive pulmonary disease, stroke, erectile dysfunction (ED), depression, cognitive impairment, Alzheimer disease (AD) and cancer-related death. Recent data suggests that COVID-19 may adversely affect OSA and that treated OSA patients may be at increased risk of death from COVID-19.

While not all OSA patients are obese, undoubtedly excess weight is as an independent causative factor to the development of OSA, with the severity of disease strongly associated with an increase in body weight ${ }^{3}$. OSA alone can promote obesity related effects further potentiating increased CV morbidity and mortality.

OSA is primarily important in the context of hypertension. Blunted nocturnal BP dipping (defined as a $<10 \%$ decrease in BP during sleep) is a common feature of sleep disturbances. OSA has been reported in approximately $40 \%$ hypertensive patients, increasing to nearly $90 \%$ in resistant hypertension patients ${ }^{5}$. OSA should rise the suspicion of secondary hypertension and should be recognized as an important risk modifier increasing CV risk estimated by the SCORE system.

OSA, resulting sleep fragmentation and intermittent hypoxia promotes metabolic disturbances and is highly associated with prevalent and incident type 2 diabetes. The J-curve phenomena on the relationship between daytime napping (i.e., excessive marker of underlying sleep disorder) and the risk of diabetes or metabolic syndrome was found, with no effect of napping below 40 minutes/day.

Severe OSA is associated with significant coronary artery plaque burden independent of traditional CV risk factors. Moderate to severe OSA increases the risk of developing NSTEMI compared to STEMI, with the risk increasing with $\mathrm{AHI}$ severity ${ }^{6}$. Patients with moderate to severe OSA demonstrate less severe cardiac injury during an acute non-fatal myocardial infarction (MI) when compared to patients without or with mild OSA, suggesting that
OSA may have a cardioprotective effect in the acute phase of MI, likely via ischemic preconditioning.

OSA and AF are strongly associated and may coexist with ED forming a clinical OSAFED syndrome that considerably potentiated the global CV risk. OSA promotes AF via acute apnoea-related arrhythmogenic changes during sleep and longterm atrial remodelling. OSA is highly prevalent in AF patients referred for cardioversion?. However, most of AF patients present no symptoms of EDS, indicating that this marker is not a reliable indication for occult OSA. Further relevant findings are related to night-to-night variability in OSA severity (which cannot be detected over one sleep study) demonstrating that OSA severity on a specific night directly corresponds to AF incidence on the same day ${ }^{8}$.

Patients with OSA present with a higher prevalence of sleep-related bradycardia and conduction delay, primarily during apnoeic episodes. Undiagnosed OSA is common in patients referred for pacemaker implantation to treat nocturnal bradycardia which in addition to prolong sinus pauses and atrioventricular conduction are substantially reduced following OSA therapy with no need for pacemaker. In this context the 2018 ACC/ AHA/HRS Guidelines highlighted OSA evaluation and treatment in patients with documented or suspected bradycardia or conduction delays.

SDB plays a critical role in the development and worsening of $\mathrm{HF}$, and its prognosis ${ }^{9}$. OSA more commonly occurs in HF preserved ejection fraction $(E F)$, whereas central sleep apnoea and cyclic episodes of hypopnea-apnoea breathing (CheyneStokes) increasingly characterize patients with HF reduced EF contributing to arrhythmias and $S C D$. Thus, screening for SDB in HF patients is urgently required.

OSA is highly prevalent in patients with cerebral infarction, transient ischemic attack (TIA), ischemic stroke or haemorrhagic stroke, reaching $62 \%$ in patients with $\mathrm{AHI}>10$ events per hour and 30\% in severe OSA, and is associated with increased poststroke mortality. SDB often precedes acute stroke and TIA. Clinically relevant are findings showing that stroke and TIA patients presents specific phenotype of OSA such as significantly shorter apnoeas and hypopneas ${ }^{10}$, suggesting that optimal therapeutic approach requires routine OSA screening in patients with cerebrovascular disease even in the absence of a previous OSA diagnosis. 
The link between OSA and increased risk of dementia and $A D$ is of clinical importance. OSA may impair amyloid- $\beta$ clearance and affect the link between slow wave activity and amyloid- $\beta$.

Awareness of OSA has increased over time but still remains low. Early detection and identification of patients with SDB is integral to curb the disease burden as its CV consequences impose a large economic burden on the global healthcare system. OSA and CV disease commonly coexist sharing multiple risk factors. Thus, the implementation of the AHA Life's Simple 7 recommendations (i.e., not smoking, having a healthy diet habits, being physically active, losing excess weight, controlling cholesterol, glucose and BP levels) are vital for ideal $\mathrm{CV}$ health and can prevent from OSA mediated CV disease.
Figure 1a

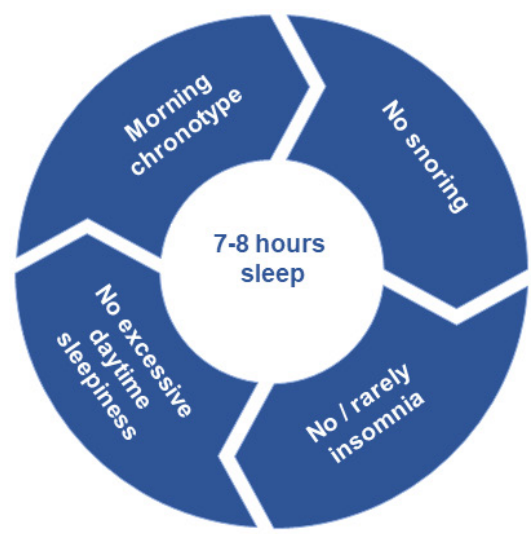

Figure 1a. Healthy sleep pattern.

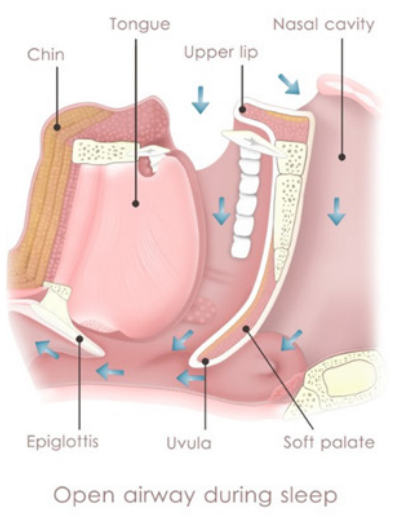

Figure $\mathbf{1 b}$. Airway during normal sleep and obstructive sleep apnoea (republished with permission

Table

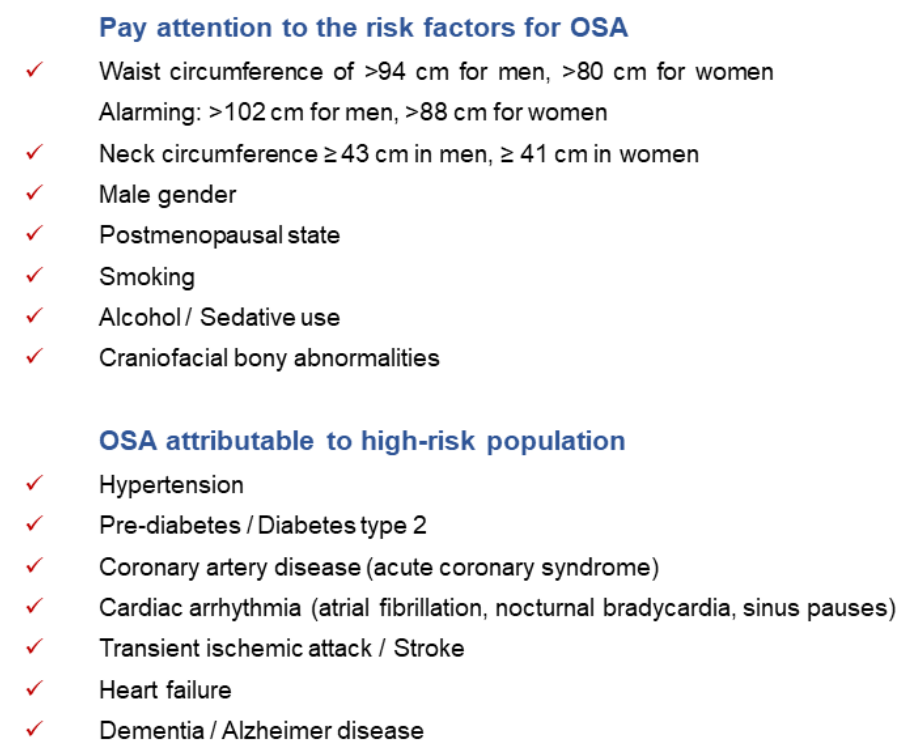

$\begin{array}{ll} & \text { OSA nighttime symptoms } \\ \checkmark & \text { Loud snoring } \\ \checkmark & \text { Often insomnia } \\ \checkmark & \text { Witnessed apneas } \\ \checkmark & \text { Nocturia } \\ \checkmark & \text { Reflux symptoms } \\ & \\ & \quad \text { OSA daytime symptoms } \\ \checkmark & \text { Excessive daytime sleepiness } \\ \checkmark & \text { Dry mouth } \\ \checkmark & \text { Morning headache } \\ \checkmark & \text { Elevated blood pressure } \\ \checkmark & \text { Difficulty concentrating } \\ \checkmark & \text { Depression, irritability } \\ \checkmark & \text { Decreased libido/ Erectile dysfunction } \\ \checkmark & \text { Cognitive dysfunction }\end{array}$

Table. Common symptoms, risk factors and high-risk population associated with obstructive sleep apnoea (OSA). 


\section{References}

1. Fan M, Sun D, Zhou T, Heianza Y, LV J, Li L, Qi L: Sleep patterns, genetic susceptibility, and incident cardiovascular disease: a prospective study of 385292 UK biobank participants. Eur Heart J 2020;41:1182-1189. DOI: 10.1093/eurheartj/ ehz849

2.Li X, Xue Q, Wang M, Zhou T, Ma H, Heianza Y, Qi L: Adherence to a Healthy Sleep Pattern and Incident Heart Failure: A Prospective Study of 408802 UK Biobank Participants. Circulation 2021;143:97-99. DOI: 10.1161/CIRCULATIONAHA.120.050792

3.Hering D, Wolf J, Chrostowska M, Narkiewicz K: Obesity and Obstructive Sleep Apnoea. In: Mancia G, Grassi G, Tsioufis KP, Dominiczak AF Rosei EA, editors. Manual of Hypertension of the European Society of Hypertension. CRC Press, 2019. DOI:10.1201/9780429199189-19

4. Benjafield AV, Ayas NT, Eastwood PR, Heinzer R, Ip MSM, Morrell MJ, Nunez CM, Patel SR, Penzel T, Pepin JL, Peppard PE, Sinha S, Tufik S, Valentine K' Malhotra A: Estimation of the global prevalence and burden of obstructive sleep apnoea: a literaturebased analysis. Lancet Respir Med 2019;7:687-698. DOl: 10.1016/S2213-2600(19)30198-5

5. Lombardi C, Pengo MF, Parati G: Systemic hypertension in obstructive sleep apnea. J Thorac Dis 2018;10:S4231-S4243. DOl: 10.21037/ jtd.2018.12.57
6. Ludka O, Stepanova R, Sert-Kuniyoshi F, Spinar J, Somers VK, Kara T: Differential likelihood of NSTEMI vs STEMI in patients with sleep apnea. Int J Cardiol 2017;248:64-68. DOI: 10.1016/j.ijcard.2017.06.034

7. Albuquerque FN, Calvin AD, Sert Kuniyoshi FH, Konecny T, Lopez-jimenez F, Pressman GS, Kara T, Friedman P, Ammash N, Somers VK, Caples SM: Sleep-disordered breathing and excessive daytime sleepiness in patients with atrial fibrillation. Chest 2012;141:967-973. DOI: 10.1378/chest.11-0975

8.Linz D, Brooks AG, Elliott AD, Nalliah CJ, Hendriks JML, Middeldorp ME, Gallagher C, Mahajan R, Kalman JM, McEvoy RD, Lau DH, Sanders P: Variability of Sleep Apnea Severity and Risk of Atrial Fibrillation: The VARIOSA-AF Study. JACC Clin Electrophysiol 2019;5:692-701. DOI: 10.1016/j.jacep.2019.03.005

9.Lachowska K, Gruchala M, Narkiewicz K, Hering D: Sympathetic Activation in Chronic Heart Failure: Potential Benefits of Interventional Therapies. Curr Hypertens Rep 2016;18:51. DOI: 10.1007/ s11906-016-0660-7

10.Leino A, Westeren-Punnonen S, Toyras J, Myllymaa S, Leppanen T, Yla-Herttuala S, MurajaMurro A, Kantanen AM, Autere J, Jakala P, Mervaala E, Myllymaa K: Acute stroke and TIA patients have specific polygraphic features of obstructive sleep apnea. Sleep Breath 2020;24:1495-1505. DOl: $10.1007 / \mathrm{s} 11325-019-02010-2$

\section{Pathophysiology of Sleep Disordered Breathing}

\section{JONAS SPAAK}

Karolinska Institutet, Department of Clinical Sciences, Danderyd Hospital, Division of Cardiovascular Medicine; and Danderyd University Hospital, Department of Cardiology, Stockholm, Sweden.

DOI:10.30824/2106-14

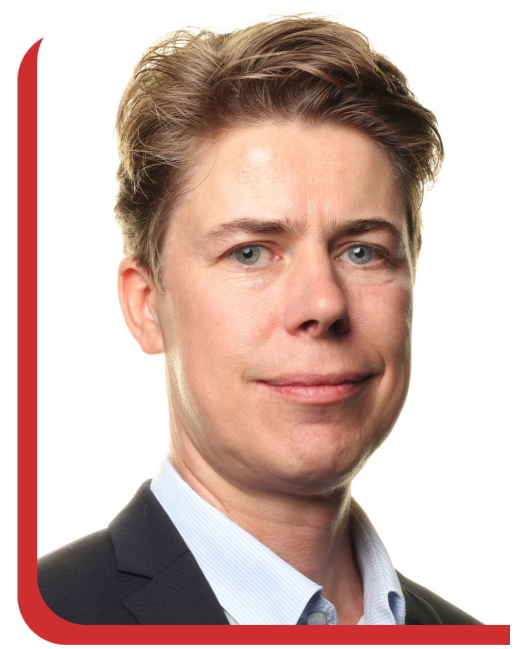

\section{Introduction}

Twenty years ago, it would have been easy to summarise the pathophysiology of sleep disordered breathing as repetitive nocturnal apnoea in obese persons causing sympathetic nerve system activation, hypertension and over time general cardiovascular disease, heart failure and stroke. The continuing studies in the field have added considerable knowledge but also many exceptions to this simplified description. Furthermore, despite the wealth of physiological understanding, the evidence for best treatment beyond weight loss and life-style modifications is limited. Part of the explanation for this is that most trials have excluded symptomatic patients, as it has been considered unethical not to offer them treatment. Nonetheless the strong association between sleep disordered breathing and obstructive sleep apnoea (OSA) in particular, 